\title{
Domain formation on oxidized graphene
}

\author{
M. Topsakal ${ }^{1,2}$ and S. Ciraci ${ }^{1,2,3, *}$ \\ ${ }^{1}$ UNAM-National Nanotechnology Research Center, Bilkent University, 06800 Ankara, Turkey \\ ${ }^{2}$ Institute of Materials Science and Nanotechnology, Bilkent University, Ankara 06800, Turkey \\ ${ }^{3}$ Department of Physics, Bilkent University, Ankara 06800, Turkey
}

(Dated: June 10, 2021)

\begin{abstract}
Using first-principles calculations within density functional theory we demonstrate that the adsorption of single oxygen atom results in significant electron transfer from graphene to oxygen. This strongly disturbs the charge landscape of the $\mathrm{C}-\mathrm{C}$ bonds at the proximity. Additional oxygen atoms adsorbing to graphene prefer always the $\mathrm{C}-\mathrm{C}$ bonds having highest charge density and consequently they have tendency to form domain structure. While oxygen adsorption to one side of graphene ends with significant buckling, the adsorption to both sides with similar domain pattern is favored. The binding energy displays an oscillatory variation and the band gap widens with increasing oxygen coverage. While a single oxygen atom migrates over the $\mathrm{C}-\mathrm{C}$ bonds on graphene surface, a repulsive interaction prevents two oxygen adatoms from forming an oxygen molecule. Our first-principles study together with finite temperature ab-initio molecular dynamics calculations concludes that oxygen adatoms on graphene cannot desorb easily without influence of external agents.
\end{abstract}

PACS numbers: 61.48.Gh,81.16.Pr,61.50.Ah

\section{INTRODUCTION}

Graphene, strictly two dimensional allotrope of carbon atom with its unique mechanical $1^{1}$, structura ${ }^{2}$, electronic $\stackrel{314}{5}$ and thermal properties, $\frac{5}{5}$ has been considered as a promising candidate for next generation electronic devices and numerous nanoscale applications. Ingenious methods have been proposed for its production ${ }^{6}[11$ Intensive studies have been also carried out for controlling and modifying various properties of bare graphene. The adsorption of foreign atoms or molecules on bare graphene surface has been considered as an efficient method to attain this objective.

Graphene oxide (GOX) is an example $e^{\sqrt{12 \mid 13}}$ to show how the properties of graphene can be changed dramatically upon the adsorption of oxygen atoms. GOX is obtained through oxidative exfoliation of graphite, which can be visualized as an individual sheet of graphene decorated with epoxy (C-O-C) and hydroxyl $(\mathrm{C}-\mathrm{OH})$ groups on both sides and edges. Incidentally, GOX has been also an attractive material for large scale graphene production 14 due to low-cost, simple and high yield reduction methods. Unfortunately, despite the oxidized graphite is a known material since last 150 years $\frac{\sqrt{15}}{}$ and great deal of experimental and theoretical research carried out recently, a thorough understanding regarding the interaction of oxygen with graphene and relevant reactions are not available yet due their stochastic nature.

To understand the experimental data, various structural configurations of GOX have been proposed based on first principles calculations. Performing the analysis of various coverage models, Boukhalov et al ${ }^{[17}$ revealed that $100 \%$ coverage of GOX is energetically less favorable than $75 \%$ coverage. Also, while a coverage less than $25 \%$ of GOX contains only hydroxyl groups, the mixed GOX consisting of both oxygen and hydroxyl is favored for higher coverage. In a later study, Yan et al! $!^{18}$ suggested that it is energetically favorable for the epoxy and hydroxy groups to aggregate together to form specific types of strips with $s p^{2}$ carbon regions in between. In contrast, Wang et al $l^{21}$ argued that thermodynamically stable structures are fully covered without any $s p^{2}$ carbon. The domains of graphene monoxide with $N_{O} / N_{C}=1$ (i.e. the ratio of number of oxygen $N_{O}$ to the number of carbon atoms $N_{C}$ ) is attained by the oxidation of both sides ${ }^{24}$ Very recent study ${ }^{25}$ combining experimental results and first principles calculations shows that multilayer GOX is metastable at room temperature undergoing modifications and reduction with a relaxation time of approximately 35 days. At the quasi-equilibrium, the nearly stable oxygen coverage was reported as $\Theta=0.38$ and presence of $\mathrm{C}-\mathrm{H}$ species is found to favor the reduction of epoxides and to a lesser extent hydroxyl groups with the formation and release of water molecules.25

From our point of view, there exists still controversies between theory and experiment. For example, yet the distribution of hydroxy and epoxy groups on GOX surface together with the trends related with their clustering or uniform coverage are unknown. At least, a rigorous explanation for the reason of the differences in the interpretations of experimental data is required. In particular, it is not clear why the desorption of oxygen adatoms through $\mathrm{O}_{2}$ formation does not occur so easily despite the negative formation energy of oxygen adsorption. Unlike GOX, the hydrogenated graphene, i.e. graphane $(\mathrm{CH})^{26 \mid 27}$ and fluorinated graphene, i.e. fluorographene $(\mathrm{CF})^{28129}$ are experimentally realized and their crystal structure are well understood.

In this study we present an extensive analysis of the oxygen adsorption and oxygen coverage by using first principles calculations based on Density Functional Theory (DFT). In order to understand the reversible oxidation-deoxidation processes ${ }^{13130}$ we consider only oxygen adatoms on graphene surfaces, in spite of the 

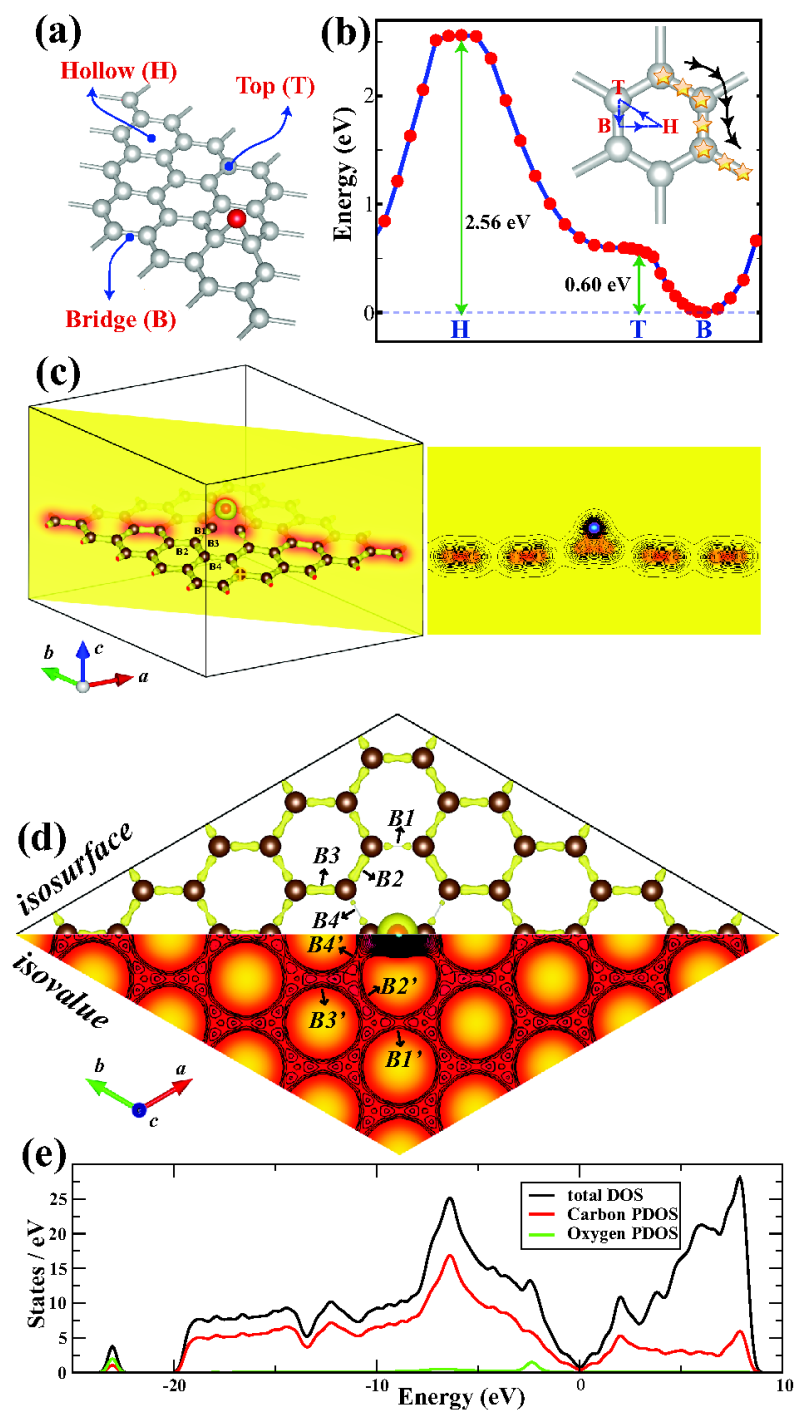

Figure 1: Various critical sites of adsorption on the 2D honeycomb structure of graphene and an oxygen atom adsorbed on the bridge site, which is found to be as the energetically most favorable site. Carbon and oxygen atoms are shown by gray and red balls, respectively. (b) Variation of energy of oxygen adatom adsorbed to graphene along $H \rightarrow T \rightarrow B$ directions of the hexagon. The diffusion path of a single oxygen adatom is shown by stars. (c) Charge density isosurfaces, isovalues and contour plots of oxygen adsorbed graphene in a plane passing through C-O-C atoms. (d) Same as (c) on the lateral plane of honeycomb structure. (e) Total and partial density of states projected to carbon and oxygen atoms. Calculations are carried out for supercell presented in (c) where $\mathrm{O}-\mathrm{O}$ interaction is significantly small.

fact that hydroxyl groups are readily coadsorbed. Earlier studies have followed approaches, which consider the optimized geometries corresponding to the minimum of total energy. Here, we show that the mechanism of oxygen coverage is governed mainly by the charge density profile of graphene, which is modified by each adsorbed oxygen in the course of oxidation. At the end, oxidized regions of graphene tend to form domains instead of a uniform coverage. In view of these results we also discuss unzipping process of graphene. $\frac{1423}{123}$ The oxygen adsorption on both sides of graphene was shown to be energetically more favorable than the adsorption to only one side, whereby serious distortions of the graphene lattice occurred. The repulsive interaction between two oxygen adatoms at the close proximity is repulsive and hinders oxygen desorption through $\mathrm{O}_{2}$ formation. We finally showed that the distribution of oxygen atoms on graphene affects the electronic properties. Even if the massless Dirac-fermion behavior of graphene can be recovered for patterns conserving specific symmetries, the band gap normally increases with increasing non-uniform oxygen coverage and attains the value as high as $3 \mathrm{eV}$. These results are critical for the device applications based on reversible oxidationdeoxidation of graphene surfaces $\frac{13130}{130}$

\section{METHOD}

Calculations are carried out within spin-polarized and spin-unpolarized density-functional theory (DFT) using projector augmented wave (PAW) potentials ${ }^{33}$ The numerical calculations have been performed by using VASP package .3132 The exchange correlation potential is approximated by generalized gradient approximation functional of Perdew, Burke, and Ernzerhof (PBE) ${ }^{34}$ Calculations are carried out using periodically repeating supercell geometry, where the spacings between graphene layers are taken $15 \AA$. However, systems involving very large graphene sheets are treated with $10 \AA$ spacing, which is still large and hinders interlayer coupling. A plane-wave basis set with kinetic energy cutoff of 500 $\mathrm{eV}$ is used. All atomic positions and lattice constants are optimized by using the conjugate gradient method, where the total energy and atomic forces are minimized. The convergence for energy is chosen as $10^{-5} \mathrm{eV}$ between two steps. Oxygen-adatom and graphene system breaks inversion symmetry and a net electric-dipole moment is generated perpendicular to the graphene surface. Dipole corrections ${ }^{35}$ are applied in order to remove spurious dipole interactions between periodic images. The $\Gamma$-point i.e. $\mathbf{k}=0$ is used for rectangular supercells containing 128 carbon atoms and oxygen adatoms, while $18 \times 18 \times 1$ k-point sampling is used for primitive unit-cell. The Gaussian smearing with a width of $0.1 \mathrm{eV}$ is used in the occupation of electronic energy bands.

\section{INTERACTION OF OXYGEN ATOM WITH GRAPHENE}

A thorough analysis of the interaction of single $\mathrm{O}$ atom with graphene is essential to understand the oxidation process. Here the adsorption of single (isolated) oxygen on graphene is represented using large supercells, where $\mathrm{O}-\mathrm{O}$ interaction is minimized. Owing to its hexagonal 
crystal structure, there are three major sites for foreign atom adsorption on graphene as shown in Fig. 1 (a). The hollow $(\mathrm{H})$ site is above the center of hexagonal rings formed by carbon atoms. The top (T) site lies on top of the carbon atoms and the bridge (B) site is above the middle of each bonds connecting two adjacent carbon atoms. The bridge site is found to be most favorable adsorption site for an oxygen atom. Earlier LDA calculations predicted also B-site as energetically favorable site ${ }^{36}$ The variation of the total energy along $\mathrm{H} \rightarrow \mathrm{T} \rightarrow$ $\mathrm{B}$ sites is presented in Fig. 1 (b). The energy barrier is $0.6 \mathrm{eV}$ for an $\mathrm{O}$ atom diffusing from bridge to top site and the energy difference between bridge and hollow site is as high as $2.56 \mathrm{eV}$. Therefore, the migration paths of oxygen adatom with minimum energy barrier follow the honeycomb structure over the $\mathrm{C}-\mathrm{C}$ bonds by going from B- to T-sites as illustrated by inset in Fig. 11(b). On the other hand, the energy barrier against the penetration of an oxygen adatom from one side of graphene to the other side is as high as $6 \mathrm{eV} \stackrel{39}{ }$ This high energy barrier suggests that graphene can be used an ideal coating preventing surfaces from oxidation. We note that the hollow site of graphene is more favorable for other atoms ${ }^{37 / 38}$ such as $\mathrm{Li}$ or $\mathrm{Ti}$, while $\mathrm{H}$ and $\mathrm{F}$ atoms prefers the top site for adsorption. 27/29

The binding energy of oxygen on graphene is defined as

$$
E_{b}=E_{T}[G r]+E_{T}[O]-E_{T}[G r+O]
$$

where $E_{T}[G r+O], E_{T}[G r], E_{T}[O]$ denote the optimized total energies of graphene with adsorbed oxygen, pristine graphene and free $\mathrm{O}$ atom, respectively. Our calculations show that $E_{b}=2.35 \mathrm{eV}$ for the $(2 \times 2)$ graphene supercell containing 8 carbon and one oxygen atom, but it increases to 2.40 for $(3 \times 3)$ and to 2.43 for $(4 \times 4)$ supercells. For supercells larger than $(4 \mathrm{x} 4)$, which correspond to smaller oxygen coverage and hinders $\mathrm{O}-\mathrm{O}$ coupling, the binding energy does not change and mimics the binding energy of single, isolated oxygen attached to graphene surface. The calculated binding energy for full coverage $\Theta=0.5$ (namely the ratio, $N_{C}, N_{O} / N_{C}=0.5$ ) is $2.80(3.34$ ) $\mathrm{eV}$ per oxygen atom for one-sided (two-sided) adsorption. The binding energy of single oxygen adatom increasing from $2.43 \mathrm{eV}$ to $2.80 \mathrm{eV}$ at full coverage indicates a significant O-O coupling. We note that the formation energy $E_{f}=E_{b}-E_{b, O_{2}} / 2$, where $E_{b, O_{2}}$ is the binding energy of $\mathrm{O}_{2}$ molecule) is negative for one-sided coverage indicates instability. However, this situation does not impose desorption of $\mathrm{O}$ through $\mathrm{O}_{2}$ formation for reasons discussed in Sec. V.

According to the Pauling scale, oxygen has an electronegativity of 3.44 , which is the second highest in periodic table after fluorine (3.98) and hence the oxidation of graphene is expected to result in significant charge transfer between oxygen and carbon atoms. Our calculations using Bader analysis ${ }^{40}$ estimates a charge transfer of 0.79 electrons from carbon atoms of graphene to oxy- gen. This charge is mainly transferred from the nearest two carbon atoms forming the bond above which the oxygen adsorbed at bridge site, while some nearby oxygen atoms also contribute to the charge transfer. Figure 1 (c) shows isosurface and isovalue (contour) plots of total charge for a plane passing through carbon atoms and oxygen. The direction of electron density increasing from carbon atoms towards oxygen atom is a clear indication of charge transfer. In addition, two carbon atoms below oxygen are slightly raised from the plane of other carbon atoms and the charge distribution of the bond between them is disturbed.

In Fig. 1 (d) bird's-eye view of isosurface and isovalue of charge density profile of a single oxygen adsorbed to each $(5 \times 5)$ supercell of graphene is presented. The structure is symmetric and the oxygen atom is at the center. We label some of the bonds corresponding to nearby bridge sites as B1,B2,B3,B4 and denote their equivalent sites by primes. While the isosurface plots of all $\mathrm{C}-\mathrm{C}$ bonds of bare graphene are identical, the adsorption of single oxygen modifies the charge distribution at its close proximity. In fact, the isosurfaces plotted for 0.3 (electron $\left./ \AA^{3}\right)^{41}$ show that the electron population of specific bonds are higher. As clearly seen from the figure, B2 and B3 bonds contain more electrons than in B1 and B4 bonds. The reason for the electron depletion in these bonds is related with the donation of electrons from these bonds to adsorbed oxygen. Interestingly, the bonds B2, B3 and their four images contain more electron density compared to B1 and other bonds further away from the oxygen atom. For a better illustration of bond charge alternation, we also presented the isovalue plot of total charge density in the upper triangle of Fig. 1 (d). Again, the more isolines in the isovalue map corresponds to more charge at B2' and B3' compared to B1' and B4'. In this context, we note that the long range interactions and Friedel oscillations found in 1D carbon chair ${ }^{42}$ and $2 \mathrm{D}$ graphene ${ }^{43}$ induced by adatoms. Finally we include the density of states (DOS) plot in Fig. 1 (e) for the system presented in Fig. 1 (a). The overall total DOS represents a profile similar to bare graphene DOS making a dip near the Fermi level corresponding to Dirac points and DOS projected to oxygen atom is represented by a peak around $-2.5 \mathrm{eV}$. Later we show that the electronic density and band gap will change with oxygen coverage.

\section{A. Interaction of Single Oxygen Molecule $\left(\mathrm{O}_{2}\right)$ with Graphene}

In contrast to oxygen atom, an oxygen molecule has a weak binding with graphene. We calculated its binding energy to be $115 \mathrm{meV}$ which consists of $57 \mathrm{meV}$ Van der Waals interaction 44 and $58 \mathrm{meV}$ chemical interaction. Its magnetic moment is $1.90 \mu_{B}$, slightly smaller than the magnetic moment of free $\mathrm{O}_{2}$ due to weak chemical interaction. The $\mathrm{O}_{2}$ molecule lies parallel, approximately 3 
$\AA$ above the graphene plane, and do not induce any distortions to graphene honeycomb structure as in the case of single oxygen adsorption. Accordingly, the binding of $\mathrm{O}_{2}$ to graphene is specified as physisorption. It is therefore concluded that graphene cannot be oxidized directly by $\mathrm{O}_{2}$ molecule unless its dissociation into oxygen atoms takes places at the vacancy site. 39

\section{COVERAGE OF GRAPHENE SURFACE WITH OXYGEN ATOMS}

\section{A. Coverage of oxygen on one side}

Starting from single oxygen adatom, we next consider the adsorption of more oxygen atoms one at a time on graphene surface leading to higher coverage of oxygen. We exclude the hydroxyl groups in the present study to simplify the situation and hence to reveal essential aspects of oxygen adsorption. In order to reduce the effects of cell size, we construct a larger rectangular supercell containing 128 carbon atoms as shown in Fig. 2 (a). The isosurface charge density profile for rectangular supercell is similar to the charge density profiles in Fig. 1 (d) with $B_{1-2}$ and $B_{1-3}$ bonds having more charge compared to $B_{1-1}$ and $B_{1-4}$. For the adsorption of second oxygen, we try all inequivalent bridge sites and calculate their total energies. It turns out that, the energetically most favorable site for the second oxygen adsorption is at $B_{1-2}$ site in Fig. 2 (a). In addition, the calculated binding energy of the second oxygen is around $2.9 \mathrm{eV}$ and this is even higher than the binding energy of single oxygen on graphene. The binding energy is $154 \mathrm{meV}$ lower for adsorption on $B_{1-3}$. The binding energy at $B_{1-5}$ site is equal to the single oxygen binding energy. But interestingly, $B_{1-1}$ and $B_{1-4}$ sites are energetically less favorable sites for second oxygen adsorption compared to other sites. The calculated $E_{b}$ is $2.33 \mathrm{eV}$ for $B_{1-1}$ site. These calculated energies indicate a direct correlation between the binding energies and isosurface profiles given in Fig. 2 (a). Apparently an oxygen atom prefers the bridge sites, where the electron density is highest compared to other available sites.

The charge density isosurface profile of graphene supercell containing two oxygen atoms is presented in Fig. 2 (b) and this profile can be used to predict the energetically favorable and unfavorable sites for the adsorption of third oxygen. Again, there are some bridge sites such as $B_{2-1}$ and $B_{2-2}$ containing more electronic charge compared to other bonds like $B_{2-3}$ and $B_{2-4}$. The third oxygen is bound to $B_{2-1}$ site with $E_{b}=3.06 \mathrm{eV}$ which is slightly higher for the maximum binding energy of second oxygen. The binding energies at $B_{2-3}$ and $B_{2-4}$ are approximately $0.7 \mathrm{eV}$ smaller than the binding energy at $B_{2-1}$ site. For the case of fourth oxygen, $B_{3-2}$ site in Fig. 2 (c) having more bond charge compared to other sites is energetically most favorable. It's binding energy, $E_{b}=2.90 \mathrm{eV}$, is slightly smaller than the binding energy of previous oxygens. The favorable binding energy for fifth oxygen can be predicted as $B_{4-1}$ from Fig. 2 (c).

The oxidation process of graphene for more than four oxygen is presented in Fig. 2 (e) and (f) up to 12 oxygens adatoms. The main trend is that each oxygen added to system prefers the bridge sites containing higher bond charge. For the sake of comparison, we included the ordered configuration for 12 adatoms in Fig. 2 (f). However, this configuration (right) is significantly less energetic, by $1.46 \mathrm{eV}$ compared to to the random configuration on the left. We continue to examine the growth of the domain consisting of 12 atoms by adding oxygen atoms to the system. The $13^{\text {th }}$ oxygen inserted to the system (not shown in figure) prefers the bridge site on the bond having highest electronic charge, but not the third bridge sites stacking eventually three oxygen atoms along a line of bridge sites of consecutive parallel C-C bonds. There are two such possible sites in Fig. 2, which are identified as the precursors of unzipping (where the usual angle of $\mathrm{C}-\mathrm{O}-\mathrm{C}$ bridge bond increases by breaking (or weakening) the C-C bond underneath, $\frac{14 \mid 23}{21}$ are energetically unfavorable by $\sim 0.9 \mathrm{eV}$. The 14 th oxygen atom behaved like the previous one: instead of occupying two possible sites of precursor states, it is adsorbed to a different bridge site which is energetically $614 \mathrm{meV}$ more favorable.

Clearly, the final structure is a domain of oxygen adatoms on graphene for $\Theta<0.5$ and hence it lacks the signatures of any uniform coverage which is present for the case of hydrogen and flourine adsorption on graphene. In the case of oxygen, adatoms arrange themselves on graphene starting from a single adatoms. Subsequently, additional ones seek energetically most favorable sites clustering around the existing ones. This domain structure The binding energies of the last adsorbed oxygen atom (or $n^{\text {th }}$ adsorbed oxygen) is calculated from the expression $E_{b}[n]=\left(E_{T}[n-1]+E_{T}[O]\right)-E_{T}[n]$ in terms of the minimum total energies of $n-1$ and $n$ oxygen atoms adsorbed on the same supercell of graphene. For any $n$, the lowest total energy (with negative sign) $E_{T}[n]$ and hence highest binding energy (with positive sign) $E_{b}[n]$ is determined by comparing the calculated energies of $n^{\text {th }}$ oxygen adatom when adsorbed to sites remained from the domain of adsorbed $(n-1)$ oxygen atoms. Once oxygen adatoms nucleate a domain they prefer to grow it by including additional oxygen atoms, whereby uniform oxidation of graphene surface is precluded. This conclusion is attained by calculating the total energy of a single domain consisting of 12 oxygen atoms formed on a large graphene supercell consisting 256 carbon atoms and comparing it with the total energy of two separate domains of 6 oxygen atoms nucleated at two different locations on the same supercell. The growth of a single domain is found to be favored by $330 \mathrm{meV}$ compared to the growth two separate domains. It should be noted that the present analysis is done under the condition, where sequential adsorption of oxygen adatoms achieved in equilibrium. However, oxidation is a stochastic process 
(a) [1 oxygen ]



(c) [3 oxygens ]

(f)

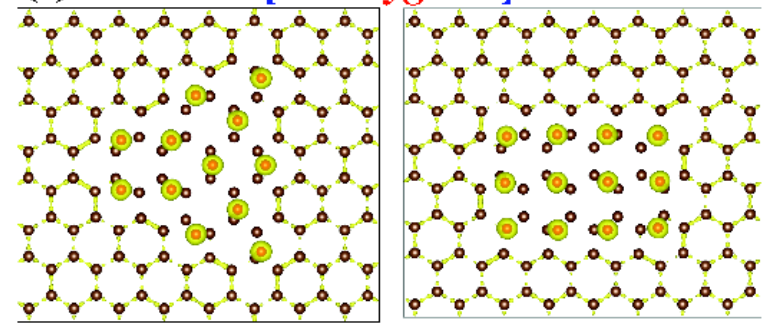

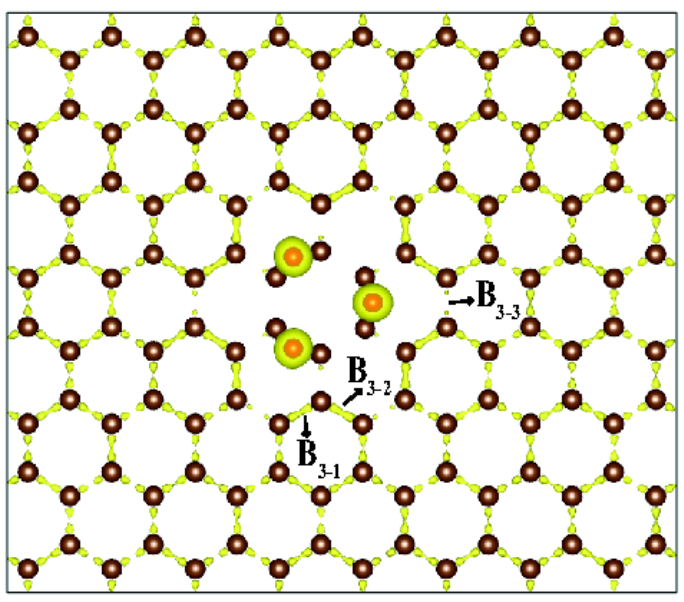

[ 12 oxygens ] (b) [ 2 oxygens ]



(d) [4 oxygens ]

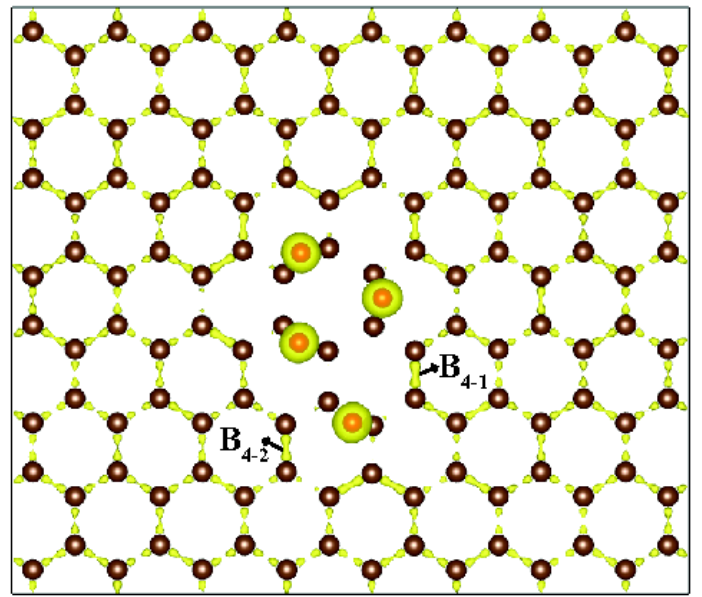

[ 9 oxygens ] (e) $[5$ oxygens ]

$0_{0}, 0_{0} 0_{0} 0_{0} 0_{0}, a_{0}$

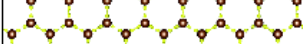

$.0 .0,0.0,0, a, a$

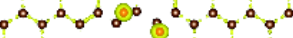

$.0,0_{0} 0,8,0,0.0 .0$

$0_{0}, 0.0,0.0,0.0$

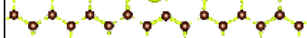

$0_{0}, 0_{0}, a_{0} 0_{0} a_{0}$,

[ 8 oxygens]

$0_{0} a_{0} a_{0} a_{0} a_{0} a_{0} a_{0} a$

$.0 .0 .0 .0,0,0,0.0$

$\therefore .0 .0 .0,0,0.0$

$0_{0} 0.0,000,0.0$

$\therefore \circ 0_{0}^{\circ} 0.0 .0$

$-0.00,0.0 .0$

$0.0 .0,0,0,0,0,0$

0.000000 (g)



Figure 2: (Color Online) (a) Charge density isosurfaces in a rectangular supercell containing 128 carbon atoms and a single adsorbed $\mathrm{O}$ atom shown by a red dot. $\mathrm{B}_{i, j}$ identifies a specific $\mathrm{C}-\mathrm{C}$ bond, where $i$ indicates the total number of oxygen atoms in the supercell and $j$ labels some of the bond around adsorbed oxygen atom(s). (b)-(c) and (d) are same as (a), except that 2,3, and 4 oxygen atoms are adsorbed to the sites, which are most favorable energetically. (e) Energetically favorable configurations up to 11 oxygen atoms adsorbed on graphene deduced from charge. (f) Energetically favorable configuration (left) and less energetic, ordered configuration (right) for $12 \mathrm{O}$ atoms. (g) Variation of the binding energies of the last oxygen adatom up to 12.

comprising processes or events taking place in nonequilibrium. Therefore, growth of multiple domains at finite temperature may occur, but the uniform growth appears to be a case of least probability.

In Fig. 2 (g), the calculated binding energy, $E_{b}[n]$ exhibits an oscillatory variation for $n>2$ and $E_{b}[n=$ $12]=3.1 \mathrm{eV}$. These oscillations are physical since energetically favorable site for the $n$th adsorbed oxygen and the resulting $E_{b}$ are well-determined and unique, but not equivalent to previous sites. The oscillations of $E_{b}$ origi- 
nate from the changes of the charge distribution and distortions of C-C bonds on the graphene surface occurred as a result of adsorbed oxygen atoms forming a domain.

At this point, we note that the energetic sites of two and three oxygen adatom in Fig. 2 is in agreement with the adsorption sites found by Yan and Chou $\frac{19}{19}$ as well as by Sun and Fabris ${ }^{23}$ However, the ground state configuration of freely adsorbed oxygen atoms forming a domain comprising four or more atoms in Fig. 2 are different from that leading to the C-C unzipping, since the latter configuration first require to overcome a significant energy barrier.23 As a matter of fact, two oxygen adsorbed to the bridge sites on two parallel C-C bonds of the same hexagon was unfavorable energetically by $1.5 \mathrm{eV} \prod^{19 \mid 23} \mathrm{On}$ the other hand, at advanced stages of Fig. 2 comprising a domain of 8 oxygen atoms we obtained a configuration consisting two B-sites occupied by oxygen atoms on the adjacent parallel C-C bonds, which can be a precursor of unzipping if subsequently adsorbed oxygen atoms occupy additional adjacent B-sites on a line and they overcome an energy barrier to increase C-O-C bond by breaking the C-C bond underneath. However, next adsorbed oxygen as well as $13^{\text {th }}$ and $14^{\text {th }}$ stopped to develop the precursor state and preferred different sites. We note that at finite temperature and in the presence of other external effects oxygen atoms are prone to develop precursors of unzipping by deviating fro above sequence of adsorption taking place in equilibrium. Later in Sec. VII we discuss this issue further. We also note that patterns of oxygen coverage for $N_{O} / N_{C}=0.5$ predicted by the genetic algorithm ${ }^{222}$ cannot be directly comparable with the nonuniform coverage in the present study, which determines the most energetic sites when oxygen atoms are adsorbed on graphene sequentially one at a time.

\section{B. High temperature behavior}

In order to test the stability of oxygen covered region in Fig. 2 (f), we also performed finite temperature, abinitio molecular dynamics calculations. The Nose thermostat is used and the time steps are taken 2.5 femtoseconds. Atomic velocities are normalized at every 40 time steps and calculations lasted for 10 picoseconds at $1000 \mathrm{~K}$ for supercell containing 128 carbon atom and 10 oxygen adatoms. At the end, this structure remained stable and neither $\mathrm{O}_{2}$ formation, nor dissociation of oxygen atoms from graphene surface did occurred. High binding energies of adsorbed oxygen atoms and absence of oxygen dissociation or any irreversible structural transition at $1000 \mathrm{~K}$ suggest that the oxygen covered domains shall remain intact for reasonable times in spite of the fact that underlying graphene is locally distorted.


Figure 3: (Color Online) Adsorption of two oxygen atoms on one surface of graphene with buckling of $0.88 \AA$. (b) Adsorption of two oxygen atoms at both sides with a buckling of 0.25 $\AA$. (c) Isosurfaces of bond charge densities after the adsorption of two oxygen atoms, each one adsorbed to different sides of graphene. Some of the C-C bonds of graphene, which are deprived of regular charges upon oxidation, are highlighted with arrows.

\section{Coverage of oxygen on both sides of graphene}

The adsorption of oxygen atoms on one side of graphene and hence formation of domain structure induces structural deformations at the underlying graphene. Fig. 3 (a) shows the side-view of graphene structure with two oxygen atoms adsorbed on the same surface of graphene. The carbon atoms below the adsorbed oxygen atoms are distorted and raised towards oxygen atoms. The resulting buckling is as large as 0.88 $\AA$ above the plane of graphene. The amount of these distortions are further increased with the addition of more oxygen atoms. In contrast to this situation, the amount of buckling is reduced to $0.25 \AA$, if the second oxygen atom were adsorbed to the other surface of graphene, as shown in Fig. 3 (b). Nonetheless, the latter configuration is $110 \mathrm{meV}$ more energetic than the previous case. This result is good in agreement with Ref[19. Hence, the two-sided adsorption shall be preferred instead of the single-sided adsorption. Despite that the favorable binding site of second oxygen atom at the other side follows our bond charge density analysis discussed in Sec. IV (B). For example, when an oxygen atom is adsorbed on 
one side as in Fig. 2 (a), the most favorable adsorption side for second oxygen is the same and is $B_{1-2}$ site no matter whether the second oxygen adsorbs to the top surface (one-sided adsorption) or to the bottom surface (two-sided adsorption). Moreover, the isosurface charge density profiles shown in Fig. 3 (c) for the case of second oxygen adsorbed on other side are identical to the profile in Fig. 2 (b) when two of the oxygens are adsorbed on one side. The ordering of energetically favorable sites presented in Fig. 2 for higher oxygen coverage is independent of the adsorption side. Nonetheless, two sided adsorption is energetically more favorable.

\section{Carbon (C) and Fluorine (F) adsorption on graphene}

We now investigate the adsorption of carbon $(\mathrm{C})$ and fluorine (F) atoms on graphene in the context of previous charge density analysis. Similar to oxygen atom, carbon atom is adsorbed at the bridge site. The atomic structure of carbon atom adsorbed on graphene presented in Fig. 4 (a) is reminiscent of the oxygen atom adsorption on graphene as presented in Fig. 1 (a). The distance between the adsorbed carbon atom and nearest-neighbor carbon atoms of graphene is $1.52 \AA$, which is slightly larger than the distance of nearest carbon-oxygen atoms $(1.46 \AA)$ in GOX. The angle formed between adsorbates and host graphene atoms which is 62.5 degree is almost equal for carbon and oxygen adsorption. On the other hand, the binding energy of carbon atom adsorbed on (5x5) supercell of graphene is $1.56 \mathrm{eV}$ and significantly smaller than the binding of oxygen adatom on graphene. The Bader analysis calculates a charge transfer of 0.04 electrons from the adsorbed carbon atom at the bridge site to the host graphene atoms and this value is also significantly smaller and is in the reverse direction as compared charge transfer between carbon and oxygen atoms. Consequently, the chemical interaction between carbon atoms is covalent rather than ionic. Nonetheless, owing to formation of new covalent bonds between $\mathrm{C}$ adatom and graphene the isosurface charge density of $\mathrm{C}-\mathrm{C}$ bonds presented in Fig. 4 (a) mimics the isosurface in the case of oxygen adsorbed on graphene as presented in Fig. 1 (d). The nearby bonds of $B_{C 1}$ and $B_{C 2}$ contain more electronic charge compared to $B_{C 3}$ and other $\mathrm{C}-\mathrm{C}$ bonds as shown in Fig. 4 (a). Moreover, it was argued that local disturbances on graphene are long ranged! $42|43| 45 \mid 46$ Interestingly, when a second carbon atom is adsorbed at the close proximity of a second carbon adatom, the bonds of the first one are broken and subsequently it is attached on top of the second adsorbed $\mathrm{C}$ atom to form $\mathrm{C}_{2}$ molecule. This way $\sim 5 \mathrm{eV}$ energy is gained. The growth of $\mathrm{C}_{n}$ atomic chain continues whenever an adsorbed carbon atom approaches the existing $\mathrm{C}_{n-1}$ chain, whereby the chain is detached from graphene and attached to the top of adsorbed carbon atom. These results confirm the earlier study on the perpendicular growth of $\mathrm{C}_{n}$ chains


Figure 4: (Color Online) (a) The bonding configuration of a single carbon atom on graphene and the resulting redistribution of bond charges shown by isosurfaces. (b) The bonding configuration of a single fluorine adatom on graphene with energetically favorable top site. The resulting charges of $\mathrm{C}-\mathrm{C}$ bonds at close proximity are shown by isosurfaces. (c) The growth pattern in the course of the fluorination of graphene.

on graphene! ${ }^{47}$ While attractive interaction between adsorbed carbon atoms on graphene give rise to the growth of chains on graphene, the repulsive interaction between oxygen adatoms hinders the formation of $\mathrm{O}_{2}$ molecules.

The atomic structure and isosurface charge density profile of fluorine atom adsorbed on graphene is presented in Fig. 4 (b). For the case of fluorine adsorption, the energetically favorable site is the top site as shown in Fig. 4 (b). The binding energy of single $\mathrm{F}$ atom adsorbed on a $(4 \times 4)$ graphene is calculated within LDA and was found to be $2.71 \mathrm{eV} \stackrel{29}{ }$ However, present calculations using $\mathrm{PBE}+\mathrm{vdW}$ correction ${ }^{44}$ yield a binding energy of 1.99 $\mathrm{eV}$ for adsorption of single $\mathrm{F}$ atom on a $(5 \times 5)$ graphene supercell. Upon fluorine adsorption, the bond charge of nearby atoms is modified as shown in isosurface profile. The Bader analysis yields a charge transfer of 0.57 electrons from carbon atoms to the adsorbed fluorine atom at the top site and this value is also significantly close to the value of charge transfer between carbon and oxygen atoms in the present study. Similar to $\mathrm{C}$ and $\mathrm{O}$ adsorption, the nearby bonds of $B_{F 1}$ and $B_{F 2}$ contain 
more electronic charge compared to $B_{F 3}$ and other C-C bonds. The nearest top site between $B_{F 1}$ and $B_{F 2}$ bonds and its other two analogues around $\mathrm{F}$ atoms contain more electronic charge and it turns out that these are energetically most favorable sites for adsorption of additional $\mathrm{F}$ atoms.

In Fig. 4 (c) we present how $\mathrm{F}$ atoms cover graphene. The second $\mathrm{F}$ atom is bonded to the top site formed by $B_{F 1}$ and $B_{F 2}$ bonds at the other side of graphene, which is most favorable site compared to to others. The third and fourth $\mathrm{F}$ atoms are also bound to other two analogues of this site. The energetics of binding structure are in complete agreement with the amount of bond charges of nearby top sites. The final arrangement containing $10 \mathrm{~F}$ atoms show a well defined pattern and further fluorination will be continuation of this pattern.

\section{OXYGEN - OXYGEN INTERACTION}

The interaction between two free oxygen atoms in vacuum is attractive and the formation of an oxygen molecule is energetically more favorable. We set the total energy to zero when the distance $d_{O-O}$ between them is $7 \AA$. Figure 5 (a) shows the variation of the energy with the distance, $d_{O-O}$, between two oxygen atoms. The energy does not vary until $d_{O-O}$ is $3.5 \AA$, but it starts to decrease as $d_{O-O}$ decreases and passes through a minimum for $d_{O-O}=1.21 \AA$. This minimum corresponds to the equilibrium bond length of $\mathrm{O}_{2}$ molecule with a binding energy of $6.67 \mathrm{eV}$. The process is exothermic and occurs without any energy barrier. However, the situation is rather different when one of the oxygen atom is adsorbed to the graphene surface and the other one is free, but approaching from above towards it. In this case, the position of free oxygen is fixed at preset heights while it is approaching, the rest of the system consisting of adsorbed oxygen and all graphene atoms are fully relaxed within conjugate gradient method. We label some of the stages by letters, A-B-C-D-E, while the two oxygens are approaching each other as shown in Fig. 5(b). The O-O coupling is initially negligible at large $d_{O-O}$ at $\mathrm{A}$, but it passes through a minimum by lowering $0.5 \mathrm{eV}$ at point $\mathrm{B}$ corresponding to $d_{O-O}=2.63 \AA$. Further decrease of $d_{O-O}$ increases the energy increases until the point $\mathrm{C}$, which is $\sim 0.5 \mathrm{eV}$ above the point $\mathrm{B}$. Beyond $\mathrm{C}$, oxygen atom flips sideways at $\mathrm{D}$. If one prevents oxygen atom from flipping by fixing its $x$ - $y$-position, but forces it towards the oxygen atom adsorbed on graphene, the adsorbed one is desorbed and two oxygen atoms form $\mathrm{O}_{2}$ molecule at E. In this exothermic process, once the barrier is overcame, the energy decreases by $\sim 3.5 \mathrm{eV}$.

For the case of two oxygen adatoms, both adsorbed on graphene and approaching towards each other, the variation of interaction energy is given in Fig. 5 (c). Some of the positions of the approaching oxygen atom on the path of minimum energy barrier are labeled by numerals. For an oxygen starting from a bridge site at I and
Oxygen - Oxygen interaction

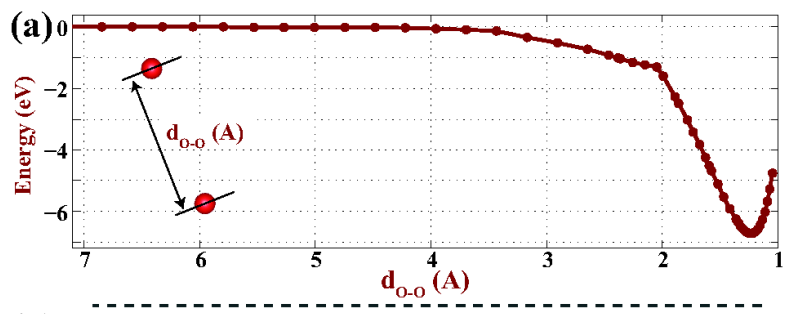

(b)
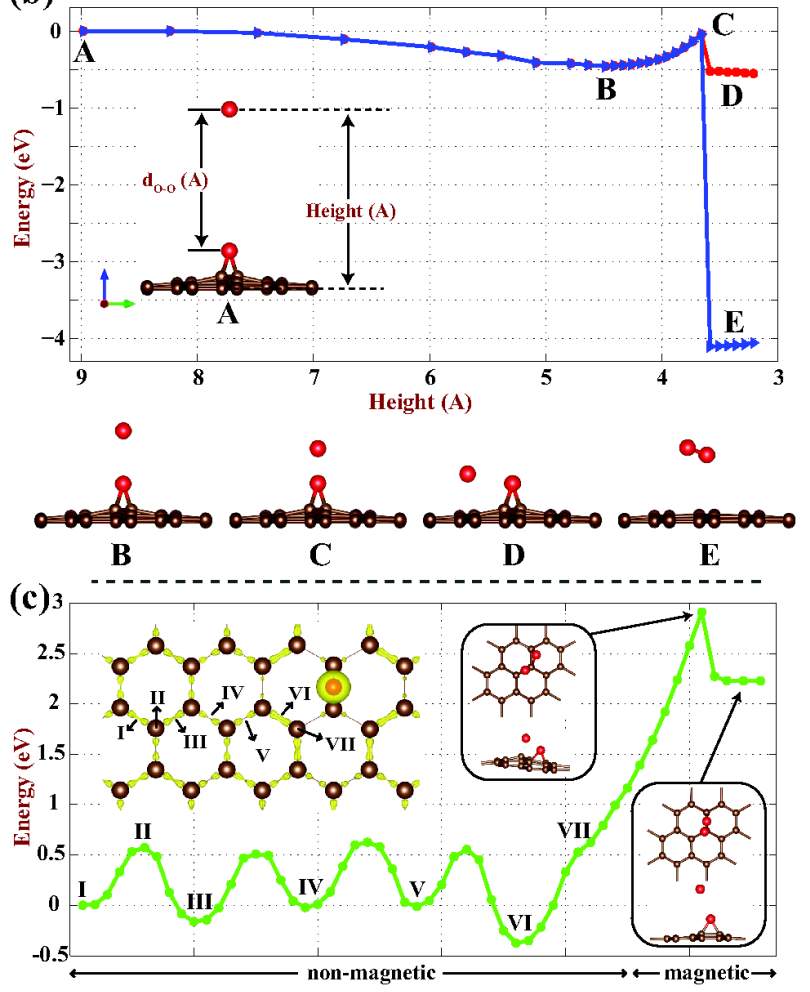

Figure 5: (Color Online) (a) The interaction energy between two free oxygen atoms approaching each other from a distance. The distance between them is $d_{O-O}$. (b) The interaction energy between a single oxygen atom adsorbed at the bridge site and a free oxygen atom approaching from the top. Different positions of approaching $\mathrm{O}$ atom are shown in the side views. (c) Variation of the energy between two oxygen adatoms on graphene. Some of the positions of the approaching oxygen atom on the path of minimum energy barrier are labeled by numerals ( I-VII ). Top and side views of the configuration of two oxygen atoms are shown by insets.

approaching towards the other oxygen, the energy shows an oscillatory behavior. The maxima, such as II, correspond to the positions where the approaching oxygen is at top site, while the minima, such as I, III, IV, V, VI correspond to positions at the bridge site. The charges of bond charge at the bridge site for reasons discussed before result in the changes in the energies at the bridge sites. For example, the bridge site VI contains more electronic charge and hence it marks the lowest energy position as one oxygen adatom is approaching the other oxygen adatom. The energetics of diffusion through the 
path between V and VI and energy barrier between them is in good agreement with the calculations by Sun and Fabris ${ }^{23}$ Since our objective is to investigate the desorption of adsorbed oxygen from graphene surface, we did not consider the energetics of diffusion from site VI to the bridge position on the $\mathrm{C}-\mathrm{C}$ bond, which is parallel to the $\mathrm{C}-\mathrm{C}$ bond holding the other oxygen. However, in Ref 23. the barrier to jump to this site is higher.

Beyond the point VII, the non-magnetic oxygen/graphene system acquires finite magnetic moments of $\approx 0.3 \mu_{B}$. Due to the repulsive interaction between two oxygen atoms adsorbed on graphene the energy increases by $\sim 3.2 \mathrm{eV}$ as shown in the Fig. 5 (c). Eventually, the approaching oxygen atom is released from the graphene when the energy barrier is overcame. The final structure is shown by inset. These results indicate that the binding energy of each oxygen on graphene is quite strong and the formation of oxygen molecule as a result of two oxygen atom approaching each other requires significant energy barrier to overcome.

\section{ELECTRONIC PROPERTIES VARYING WITH OXYGEN COVERAGE}

The electronic energy structure of GOX strongly depends on oxygen coverage, as well as on the pattern of coverage. Here we consider the electronic properties corresponding to different number of oxygen atoms adsorbed at different bridge sites of the $(4 \times 4)$ supercell repeating periodically. Bare graphene has a semimetallic electronic structure with its characteristic density of states (DOS) making a dip at the Fermi level and linearly crossing valance and conduction bands at special $\mathrm{K}$ - and $\mathrm{K}$ '-points of Brillouin Zone as shown in Fig. 6 (a). It has a zero band gap and these special symmetry points are called Dirac points 488 The energetically favorable configuration of four oxygen atoms adsorbed on a $(4 \times 4)$ hexagonal supercell is presented in Fig. 6 (b). The resulting DOS profile is different from the bare graphene, since a narrow energy gap of $70 \mathrm{meV}$ is opened. The Dirac cones disappeared and the band gap occurs at the points different than K- and K'-points. For a random and energetically less favorable distribution of oxygen atoms as in Fig. 6 (c), the energy band gap is further increased to $127 \mathrm{meV}$. The position of the minimum of conduction band and the maximum of the valence band has changed in BZ. Surprisingly, the semimetallic band structure of graphene is recovered when four oxygen atoms are uniformly disturbed on graphene surface as shown in Fig. 6 (d). Although the difference of the atomic positions from Fig. 6 (c) is minute, the band gap is closed and the density of states profile becomes similar to that of bare graphene making a dip at the Fermi level. The conduction and valance bands cross at $\mathrm{K}$ - and $\mathrm{K}$ '-special points similar to bare graphene.

Earlier, it was reported that the superstructures and nanomeshes having special point-group symmetry, which
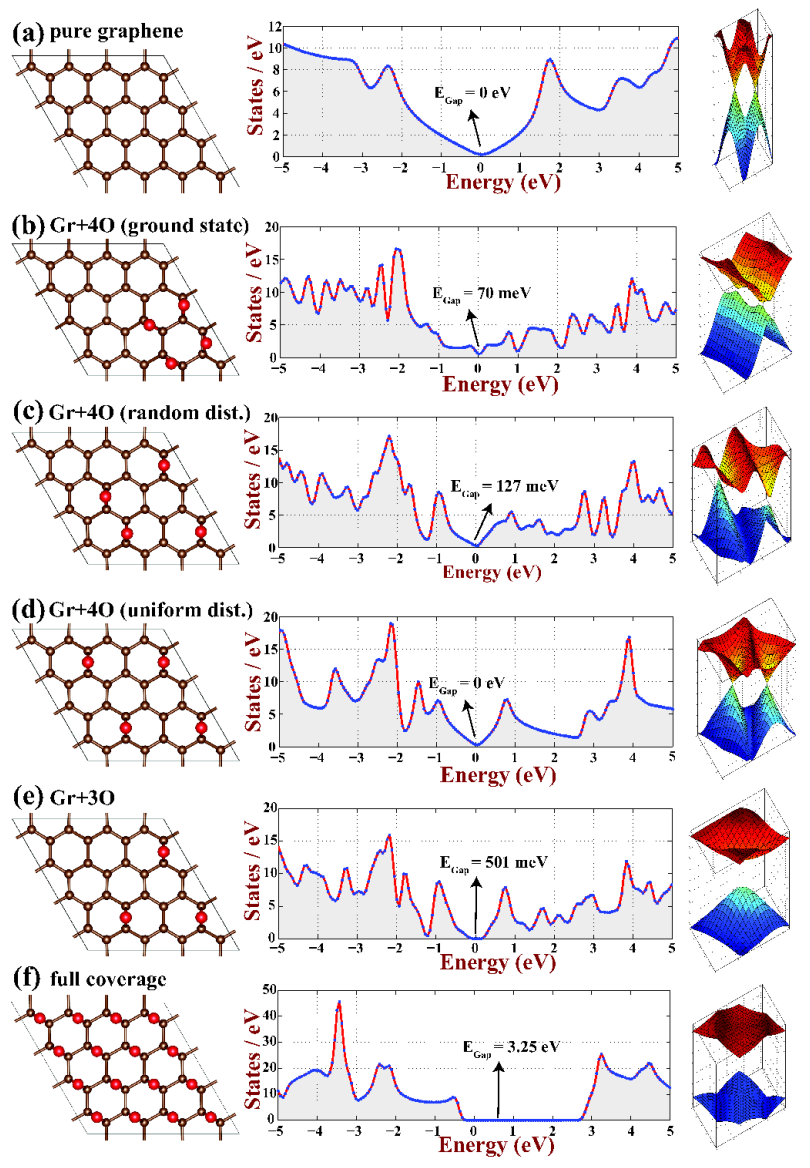

Figure 6: (Color Online) (a) Bare graphene and its typical density of states with zero state density at the Fermi level $\mathrm{E}_{F}$. The constant energy surfaces of conduction and valence bands are shown on left-hand side. (b) A four-adatom domain corresponding to lowest total energy configuration and has a band gap of $70 \mathrm{meV}$. (c) Another adsorption configuration of four oxygen adatom resulting in a band gap of $127 \mathrm{meV}$. (d) A uniform and symmetric configuration of adsorbed oxygen atoms with zero density of states at $E_{F}$. (e) A sizable band gap is opened when the symmetry of oxygen decoration is broken by the removal of a single oxygen atom. (f) The wide band gap of $3.25 \mathrm{eV}$ is opened for coverage corresponding to $\Theta=N_{O} / N_{C}=0.5$ at one side $\left(\mathrm{N}_{O}\right.$ and $\mathrm{N}_{C}$ are the numbers of oxygen and carbon atoms in the $(4 \times 4)$ supercell.

are generated by decoration of adatoms, adatom groups or holes repeating periodically in graphene matrix may give rise to the linearly crossing bands and hence to the recovery of massless Dirac Fermion behavior ${ }^{49}$ Our results in Fig. 6 (d) is a verification of this situation for oxygen adsorption on graphene. However, the perfect uniform coverage of oxygen on graphene is experimentally not achievable and we also present a situation where the periodicity of uniform coverage is broken by removal of an oxygen atom as in Fig. 6 (e). In contrast to electronic structure as in Fig. 6 (d), the Dirac behavior is completely removed and the resulting structure is semiconductor with relatively large energy band gap of 501 
$\mathrm{meV}$

For the case of $N_{O} / N_{C}=0.5$ coverage at one side, the resulting system is a wide band gap material. Fig. 6 (f) shows the atomic structure and density of states profile. Unlike bare graphene and low oxygen coverage, the resulting structure has a band gap of $3.25 \mathrm{eV}$. The two sided coverage for $N_{O} / N_{C}=0.5$ also yields similar DOS profile with a band gap wider than $3 \mathrm{eV}$. These results indicate that the regions of GOX where each carbon atom is bonded with an oxygen should be an insulator and hence should reflect light.

\section{DISCUSSIONS AND CONCLUSIONS}

Our study dealt with the adsorption of single and multiple oxygen atoms to graphene surface and explored their desorption. We showed that $\mathrm{O}_{2}$ molecule can merely be physisorbed to graphene surface. In contrast, free oxygen atoms are adsorbed at the bridge sites above C-C bonds by forming strong chemical bonds. Significant amount of charge is transferred to oxygen adatom from graphene, which disturbs the charge distribution of the $\mathrm{C}-\mathrm{C}$ bonds at the proximity of adsorbate. Additional oxygen atoms are adsorbed to the bridge sites above the C-C bonds of graphene, which has highest charge density. This behavior promotes the developments of domains of oxygen adatoms. The domain pattern which, in fact is energetically favorable is also preserved for oxygen atoms adsorbed to both sides of graphene. The binding energy of adsorbed oxygen atoms display an oscillatory change; it starts from $2.43 \mathrm{eV}$ and eventually raises to $2.80(3.34)$ $\mathrm{eV}$ at one sided (two sided) full coverage with $\Theta=0.5$. Accordingly, the formation energy of adsorbed oxygen is negative.

Even if the sequential adsorption of oxygen atoms forms domains with nonuniform coverage, full coverage can form eventually. High oxygen coverage only at one side of graphene causes to severe deformations of graphene lattice. While the adsorption configurations which can be precursors of unzipped of graphene are not favorable for low coverage of large graphene surfaces, they may occur at the edges of domains comprising large number of oxygen atoms, where underlying graphene lattice is severely distorted. Nonequilibrium conditions occurring at finite temperatures and size effects originating from the small size of underlying graphene may favor the nucleation of precursors of unzipping.

Single oxygen migrates on a pathway of minimum energy barrier of $0.6 \mathrm{eV}$ over the honeycomb structure between bridge and top sites. For the same reason the interaction between two oxygen adatoms exhibits an oscillatory variation, but becomes increasingly repulsive as the distance decreases beyond a threshold value. This repulsive interaction hinders desorption of oxygen through the formation of $\mathrm{O}_{2}$ molecule despite the negative formation energy of adsorbed oxygen atoms.

The electronic structure of oxidized graphene is strongly dependent on the coverage of oxygen and its configuration. While the massless Dirac Fermion behavior with linearly crossing bands at the Fermi level is maintained for specific coverage conserving certain rotation symmetry, the band gap opens and develops with increasing coverage of oxygen adatom. As oxidized domains dominate the surface, semimetallic bare graphene is transformed into a semiconducting material. It appears that the band gap can be engineered through oxygen coverage. Bright and dark spots observed experimentally on GOX surfaces are expected to be related with metallic and light reflecting semiconducting regions, respectively. We believe that metallic regions corresponds to $s p^{2}$-bonding regions of graphene. Our results indicate that a specific external effect is required for the fast and reversible transition between metallic and semiconducting states of graphene oxide.

\section{ACKNOWLEDGEMENTS}

This work is supported by TUBITAK through Grant No:108T234. All the computational resources have been provided by TUBITAK ULAKBIM, High Performance and Grid Computing Center (TR-Grid eInfrastructure). S. C. acknowledges the partial support of TUBA, Academy of Science of Turkey.
* Electronic address: ciraci@fen.bilkent.edu.tr

1 C. Lee, X. Wei, J. W. Kysar, J. Hone, Science 321 , 385 (2008).

2 K. S. Novoselov, A. K. Geim, S. V. Morozov, D. Jiang, Y. Zhang, S. V. Dubonos, I. V. Grigorieva, and A. A. Firsov, Science 306, 666 (2004).

3 M. I. Katsnelson, K. S. Novoselov, A. K. Geim, Nature Physics 2, 620 (2006).

4 A. K. Geim, K. S. Novoselov, Nature Materials, 6, 183 (2007).

5 A. A. Balandin, Nature Materials, 10, 569 (2011).

${ }^{6}$ C. Berger, Z. Song, X. Li, X. Wu, N. Brown, C. Naud, D.
Mayou, T. Li, J. Hass, A. N. Marchenkov, E. H. Conrad, P. N. First, W. A. de Heer, Science 312, 1191 (2006).

7 Y. Zhang, Y.-W. Tan, H. L. Stormer, P. Kim, Nature 438, 201 (2005).

8 X. Li, L. Zhang, S. Lee, H. Dai, Science 319, 1229 (2008).

9 S. Bae et al., Nature Nanotechnology 5, 574 (2010).

10 S. Tongay, M. Lemaitre, J. Fridmann, A. F. Hebard, B. P. Gila, and B. R. Appleton, Appl. Phys. Lett. 100, 073501 (2012).

11 S. Gadipelli, I. Calizo, J. Ford, G. Cheng, A.R.H. Walker and T. Yildirim, J. Mater, Chem. 21, 16057 (2011).

12 D. A. Dikin, S. Stankovich, E. J. Zimney, R. D. Piner, G. 
H. B. Dommett, G. Evmenenko, S. T. Nguyen, and R. S. Ruoff, Nature (London) 448, 457 (2007).

13 O. O. Ekiz, M. Urel, H. Guner, A. K. Mizrak, and A. Dana, ACS Nano, 5, 2475 (2011).

14 H.C. Sniepp, J-L. Li, M.C. McAllister, H. Sai, M. HerreraAlonso, D.H. Adamson, R.K. Prud'homme, R. Car, D.A. Saville, I.A. Aksay, J. Phys. Chem B, 110, 8535 (2006).

15 B. Brodie, Philos. Trans. R. Soc. London 149, 249 (1859).

16 J.-L. Li, K. N. Kudin, M. J. McAllister, R. K. Prudhomme, I. A. Aksay, and R. Car, Phys. Rev. Lett 96, 176101 (2006).

17 D. W. Boukhvalov and M. I. Katsnelson, J. Am. Chem. Soc. 130, 10697 (2008).

18 J.-A. Yan, L. Xian, and M. Y. Chou, Phys. Rev. Lett. 103, $086802(2009)$.

19 J.-A. Yan and M. Y. Chou, Phys. Rev. B 82, 125403 (2010).

20 A. Bagri, C. Mattevi, M. Acik, Y.J. Chabal,M. Chhoowalla and V.B. Shenoy, Nature Chemistry, 21, 581 (2010).

${ }^{21}$ Lu Wang, Y. Y. Sun, Kyuho Lee, D. West, Z. F. Chen, J. J. Zhao, and S. B. Zhang, Phys. Rev. B 82, 161406 (2010).

22 H.J. Xiang, S-H. Wei and X.G. Gong, Phys. Rev. B 82, 035416 (2010).

23 T. Sun, and S. Fabris, Nanoletters 12, 17-21 (2012).

24 E.C. Mattson et al., ACS Nano 12, 9710 (2011).

25 S. Kim, S. Zhou, Y. Hu, M. Acik, Y. J. Chabal, C. Berger, W. de Heer, A. Bongiorno, E. Riedo, Nature Materials 11, 544 (2012).

26 D. C. Elias, R. R. Nair, T. M. G. Mohiuddin, S. V. Morozov, P. Blake, M. P. Halsall, A. C. Ferrari, D. W. Boukhvalov, M. I. Katsnelson, A. K. Geim, and K. S. Novoselov, Science 323, 610 (2009).

27 J. O. Sofo, A. S. Chaudhari, and G. D. Barber, Phys. Rev. B 75, 153401 (2007).

${ }^{28}$ R. R. Nair, W. Ren, R. Jalil, I. Riaz, V. G. Kravets, L. Britnell, P. Blake, F. Schedin, A. S. Mayorov, S. Yuan, M. I. Katsnelson, H.-M. Cheng, W. Strupinski, L. G. Bulusheva, A. V. Okotrub, I. V. Grigorieva, A. N. Grigorenko, K. S. Novoselov, and A. K. Geim, Small 6, 2877 (2010).

29 H. Sahin, M. Topsakal, and S. Ciraci, Phys. Rev. B 83,
$115432(2011)$

30 Z. Wei, D. Wang, S. Kim, S.-Y. Kim, Y. Hu, M. K. Yakes, A. R. Laracuente, Z. Dai, S. R. Marder, C. Berger, W. P. King, W. A. de Heer, P. E. Sheehan, and E. Riedo, Science 328, 1373 (2010).

31 G. Kresse, J. Hafner, Phys. Rev. B 47, 558 (1993).

32 G. Kresse, J. Furthmüller, Phys. Rev. B 54, 11169 (1996).

33 P. E. Blöchl, Phys. Rev. B 50, 17953 (1994).

34 J. P. Perdew, K. Burke, and M. Ernzerhof, Phys. Rev. Lett. 77, 3865 (1996).

35 G. Makov and M. C. Payne, Phys. Rev. B 51, 4014 (1995).

${ }^{36}$ K. Nakada, and A. Ishii, Solid State Comm. 13, 151 (2011).

37 C. Ataca, E. Aktürk, S. Ciraci, and H. Ustunel, Appl. Phys. Lett. 93, 043123 (2008).

38 H. Sevincli, M. Topsakal, E. Durgun and S. Ciraci, Phys. Rev. B, 77, 195434 (2008).

39 M. Topsakal, H. Sahin, and S. Ciraci, Phys. Rev. B 85, 155445 (2012).

40 G. Henkelman, A. Arnaldsson, and H. Jónsson, Comput. Mater. Sci. 36, 254 (2006).

41 K. Momma and F. Izumi, J. Appl. Crystallogr. 44, 1272 (2011).

42 S. Cahangirov, M. Topsakal and S. Ciraci, Phys. Rev. B 82, 195444 (2010).

43 A. Bacsi and A. Virosztek, Phys. Rev. B 82, 193405 (2010).

44 S. Grimme, J. Comp. Chem. 27, 1787 (2006).

45 P.O. Lehtinen, A.S. Foster, A. Ayuela, A. Krasheninnikov, K. Nordlund and R.M. Nieminen, Phys. Rev. Lett. 91, 017202 (2003).

46 C. Ataca, E. Aktürk, H. Sahin and S. Ciraci, J. Appl. Phys., 106, 013704 (2011).

47 C. Ataca and S. Ciraci, Phys. Rev. B, 83, 235417 (2011).

48 K.S. Novoselov, A.K. Geim, S.V. Morozov, D.Jiang, M.I. Katsnelson, I.V. Grigorieva, S.V. Dubonos, and A.A. Firsov, Nature (London) 438, 197 (2005).

49 H. Sahin and S. Ciraci, Phys. Rev. B 84, 035452 (2011). 